\title{
Real-time multi-agent systems for telerehabilitation scenarios
}

\author{
Davide Calvaresi ${ }^{\mathrm{a}, \mathrm{c}, *}$, Mauro Marinoni ${ }^{\mathrm{a}}$, Aldo Franco Dragoni ${ }^{\mathrm{b}}$, Roger Hilfiker ${ }^{\mathrm{c}}$, \\ Michael Schumacher ${ }^{\mathrm{c}}$ \\ ${ }^{a}$ Scuola Superiore Sant'Anna, Pisa, Italy \\ ${ }^{\mathrm{b}}$ Università Politecnica delle Marche, Ancona, Italy \\ ${ }^{\mathrm{c}}$ University of Applied Science Western Switzerland (HES-SO), Sierre, Switzerland
}

\section{A R T I C L E I N F O}

\section{Keywords:}

Wearable multi-agent systems

Real-time multi-agent systems

Telerehabilitation

Real-time negotiation

Real-time scheduler

MAS

\begin{abstract}
A B S T R A C T
Telerehabilitation in older adults is most needed in the patient environments, rather than in formal ambulatories or hospitals. Supporting such practices brings significant advantages to patients, their family, formal and informal caregivers, clinicians, and researchers.

This paper presents a focus group with experts in physiotherapy and telerehabilitation, debating on the requirements, current techniques and technologies developed to facilitate and enhance the effectiveness of telerehabilitation, and the still open challenges. Particular emphasis is given to (i) the body-parts requiring the most rehabilitation, (ii) the typical environments, initial causes, and general conditions, (iii) the values and parameters to be observed, (iv) common errors and limitations of current practices and technological solutions, and (v) the envisioned and desired technological support. Consequently, it has been performed a systematic review of the state of the art, investigating what types of systems and support currently cope with telerehabilitation practices and possible matches with the outcomes of the focus group. Technological solutions based on video analysis, wearable devices, robotic support, distributed sensing, and gamified telerehabilitation are examined. Particular emphasis is given to solutions implementing agent-based approaches, analyzing and discussing strength, limitations, and future challenges. By doing so, it has been possible to relate functional requirements expressed by professional physiotherapists and researchers, with the need for extending multi-agent systems (MAS) peculiarities at the sensing level in wearable solutions establishing new research challenges. In particular, to be employed in safety-critical cyber-physical scenarios with user-sensor and sensor-sensor interactions, MAS are requested to handle timing constraints, scarcity of resources and new communication means, crucial to providing real-time feedback and coaching. Therefore, MAS pillars such as the negotiation protocol and the agent's internal scheduler have been investigated, proposing solutions to achieve the aforementioned real-time compliance.
\end{abstract}

\section{Introduction}

Healthcare institutions are facing the strain of a significantly larger older adult population [1]. A lengthening life expectancy is naturally met by an increasing demand for medical and technological contributions to extend "good-health" and disability-free period.

The major factor catalyzing the older adult impairing process is the progressive reduction of mobility and activity, and the increased susceptibility to disease, high impact falls and bone fractures [2]. Despite the emergence of less-invasive surgical techniques, post-intervention rehabilitation still requires extended periods of tailored therapies.
Performing traditional rehabilitative practices is leading to a significant increase in public-health costs and, in some cases, a lack of resources, thus worsening the quality of the services. However, implementing a method to simplify the access to health services [3] can sustain the balance between the quality of care and the growth of patient numbers. For example, patients requiring continuous or selective monitoring, can benefit from systems that automatically transmit the information gathered in their domestic environment to the health clinics, thus enabling telemonitoring of their health condition $[4,5]$. Although in traditional solutions, telemonitoring is a self-contained practice limited to passively observing the patients, the need for remote sensing is crucially

\footnotetext{
* Corresponding author at: Scuola Superiore Sant'Anna, Pisa, Italy.

E-mail addresses: davide.calvaresi@hevs.ch (D. Calvaresi), m.marinoni@sssup.it (M. Marinoni), a.f.dragoni@univpm.it (A.F. Dragoni), michael.schumacher@hevs.ch (R. Hilfiker).

URL: http://www.davidecalvaresi.it (D. Calvaresi).
} 
coupled with the need for coaching older adults in their daily living $[6,7]$. For a critical activity such as telerehabilitation, telemonitoring cannot be limited to observing patient behaviors. Indeed, patient adherence and acceptability of rehabilitative practices need to be actively enhanced, overcoming pitfalls due to motor (e.g., endurance), nonmotor (e.g., fatigue, pain, dysautonomic symptoms, and motivational), and cognitive deficits. Hence, according to Rodriguez et al. [8], telerehabilitation can be formally defined as:

"the application of telecommunication, remote sensing, operation and computing technologies, to assist with the provision of medical rehabilitation services at a distance."

Patients, physiotherapists, and health institutions can gain numerous benefits from an extensive adoption of telerehabilitation systems [9]. Considering the economical point of view, Mozaffarian et al. [10] figured out that the total cost of strokes in the US was an estimated 34.3 billion dollars in 2008, rising up to 69.1 billion dollars in 2016 .

Although the following are not precisely quantifiable due to insufficient up to date evidence [11], Mutingi et al. [12] presented "inevitable advantages" as (i) a substantial cost saving primarily due to the reduction of specialized human resources, (ii) an enhancement of patient comfort and lifestyle, and (iii) improvements of therapy and decision making processes. Moreover, Morreale et al. [13] mentioned one of the most appreciated benefits: the increase of adherence to rehabilitation protocols. The multitude of scientific contributions fostering telerehabilitation exploit new technologies and various architectures to better understand and serve user requirements. However, due to technological and/or technical limitations, physiotherapist needs have not yet been completely satisfied. To fill this gap, a system evolution is required. For example, telerehabilitation systems cannot offer the same behavior to users with diverse conditions. Viceversa, according to the environment condition, they must be able to adapt themselves to user needs [8].

Telerehabilitation is characterized by a very delicate equilibrium between environment, devices, and users. On the one hand, capabilities such as autonomic self-management, self-adaptation, extensible knowledge, flexibility, and ubiquity have been proven to be crucial to facilitate and promote usability and then the actual practices [14,15]. Hence, systems relying on a Multi-Agent approach gained increasing relevance in assistive and healthcare scenarios $[1,16,17]$.

On the other hand, the capability of providing a bounded response time (predictability) is crucial to guarantee correct feedback and safe ontime coaching. Reliability and predictability are features strongly characterizing real-time systems [18].

Therefore, to have effective and reconciling solutions, there is a need for multi-agent systems (MAS) real-time compliant or agentified real-time systems.

\subsection{Contributions}

Aiming at providing MAS capable of complying with strict timing constraints to be safely employed in the context of telerehabilitation scenarios, this paper provides the following contributions:

(i) Exploiting a focus group conducted with physiotherapists and experts in the field, it elaborates on rehabilitation requirements, practices, and activities that to date require the most technological support. Thus, it aims at raising the understanding of the current situation of the telerehabilitation field;

(ii) It performs a Systematic Literature Review (SLR) of telerehabilitation systems, dedicating particular attention to techniques and technologies, with particular emphasis on those based on multi-agent systems (MAS);

(iii) It organizes and details the requirements collected in the study mentioned in our SLR, connecting them with the needs and requirements of technological systems obtained by the study mentioned in (i).

(iv) It identifies the main challenge for MAS in telerehabilitation scenarios.

(v) It discusses the technical and technological challenges for MAS to meet the main goal identified in (iv). In particular, they concern deploying intelligent agents in distributed wearable sensor nodes while facing compliance with strict timing constraints.

In the context of envisioned MAS able to respect strict-timing constraints, named Real-Time multi-agent systems (RT-MAS), the contribution this work brings to telerehabilitation scenarios are:

- it proposes the adoption/adaption of the Constant Bandwidth Server (CBS) as the agent local scheduler (scheduling its behaviors);

- It proposes and motivates the adoption of a reservation-based negotiation protocol to fully exploit the advantages brought by the adoption of the CBS as local scheduler.

\subsection{Paper structure}

The rest of the paper is organized as follows. Section 2 presents the focus group and centers on rehabilitation needs and practices to better understand the telerehabilitation domain. Thus, it facilitates the identification and understanding of currently unmet requirements expressed by professional physiotherapists. Section 3 elaborates the Systematic Literature Review Methodology employed to rigorously study the state of the art. Section 4 provides a complete overview of the evidence collected by performing the methodology presented in Section 3. In particular, contributions from both conventional and agent-based telerehabilitation systems are presented respecting the organization of the structured research questions. Section 5 discusses the strengths and limitations of current agent-based telerehabilitation systems, introducing and detailing the future challenges to be faced by MAS to enhance performance and applicability in rehabilitation scenarios. Section 7 analyzes the telerehabilitation case study by applying contributions $(v)$, (vi), and (vii). Finally, Section 8 summarizes the lessons learnt and presents the ongoing work.

\section{Telerehabilitation: practices and requirements}

Despite the increasing awareness regarding the effectiveness of telerehabilitation, there is still a lack of high-quality studies evaluating the various types, components, modalities and duration of therapy, and the long-term functional outcomes [19].

Physiotherapists gain significant experience through education, training, and practice. However, the quality of a physiotherapy mainly relies on their experience. The absence of errors, information reproducibility, and simple knowledge sharing [20] still cannot be guaranteed.

\subsection{Focus group: description}

The study has been conducted in collaboration with the Institute of Health and its physio lab Leukerbad, where a considerable amount of practices are still carried out with conventional and non-technological methods.

Ten physiotherapists (seven women and three men) participated in a focus group [21] moderated by three researchers specializing in assistive and telerehabilitation technology. The selection of the participants was made based on their experience in physiotherapy. Among the participants, five have a Masters degree, two were Master students, and three were active researchers. All participants had at least five years of experience as physiotherapists (up to 25 years). The participants were German, French, Swedish, and Italian native speakers. However, all of them were fluently speaking and understanding English (established the language of the conversation). 
Five main topics have been covered with as many open questions, which also reflect the organization of their presentation:

- joints requiring rehabilitation, related practices and adjacent limbs involved;

- rehabilitation environment, initial causes and conditions;

- values and parameters that must/might/could be useful to observe;

- common errors and limits of patients, physiotherapists, current practices and technological solutions;

- envisioned and desired technological support.

After the focus group, the participants were invited to participate in an online survey consisting of fifteen open questions (listed in the A) covering the five topics mentioned above.

The notes elicited from the conversation have been used to refine the answers collected with the questionnaire. The outcomes are presented in the following section.

\subsection{Focus group: outcomes}

Following the study described in Section 2.1, it has been recorded that, unfortunately, both operators and patients have experienced the ease with how errors or biases can be introduced in the execution (patient's side) and measurement (physiotherapist's side) of a therapy. Moreover, the use of inadequate tools or systems only complicates the rehabilitation sessions and follow up.

By aggregating the information obtained by executing the questionnaire (A) and summarizing the outcome of the informal discussion described in the previous section, the following results are presented:

T1 - first topic

The obtained results revealed that the body parts requiring the most technological support are the knee, hip, shoulder, neck and back.

\section{T2 - second topic}

To better understand pre-surgical conditions, the rehabilitation environment, and what a system might be required to identify during a prevention phase, the most common causes generating the need for rehabilitation have been investigated. Lifestyle and aging have proven to be the most predominant causes. For example, a sedentary lifestyle might facilitate the development of arthritis/osteoarthritis and early joint degeneration, whilst an intense sportive lifestyle can cause anterior-cruciate or menisci ligament rupture and lower back pain.

\section{T3 - third topic}

The study of dynamics and physical structure evidently highlights how every body part that might require rehabilitation affects the local physical network. The crucial task would be to identify the entirety of the latter. For example, by monitoring the movements of femur and tibia, it is possible to determine the angular interval of the knee during flexion, extension, and abduction.

Summarizing the expectations expressed by the physiotherapy community, comprehensive solutions supporting the most significant rehabilitation practices are required, providing measurements if not or enhancing them to better understand:

- therapy and practice adherence;

- performance and correctness of the movements;

- possible adjustments, errors and compensations;

- coaching, encouraging and motivating the patient;

- motivation, commitment and fatigue measurements;

- specific parameters per practice (e.g., quantification of varus or valgus thrusts during gait or jumps analysis).

\section{T4 - fourth topic}

Current available solutions in the market critically lack usability and information. Devices such as the kinetec [22] help the patient's knee in performing passive and continuous movements. Such a device is usually employed twice per day for a total of two hours during the acute phase. ${ }^{1}$ However, the provided information on the knee angle is not precise due to the misalignement with the machine's angle. Such an error is mostly due to structural factors, limb misplacement or attempts to compensate the movement performed by the patient trying to reduce an undetectable pain.

The inability to identify the latter can be summarised as the system's drawback (pain, muscular resistance and patient improvement). Moreover, the use of the kinetec is supposed to be unsupervised after the initial assisted setup, thus enabling the propagation of all the aforementioned errors.

\section{T5 - fifth topic}

The "trust" in scientific research is a common element emerging in all the participants' answers confirming the belief that enhancing traditional practices with technological supports can propel patients towards a faster and better healing process. However, several functionalities are still unavailable to the market. Without any form of special commitment, the most required technological interventions are (i) quantifying the movements during rehabilitation and sport sessions, (ii) accurately measuring joint motor behavior pre-, during, and posttherapeutic intervention, (iii) qualitative assessment of the movements complementing quantitative analysis, and finally (iv) the measurement of physiological processes (e.g., cells regeneration, muscle growth and activation, blood circulation, and immune-system condition).

Finally, to better understand the concrete possibilities of technical and technological interventions, it is worth recalling that in the context of rehabilitation, a therapy is composed of activities. An activity consists of tasks which is a set of steps to be performed (e.g., gestural, postural) [23];

Considering their involvement in telerehabilitation systems, interaction tasks can be classified into four categories:

- Individual task - a task performed by a single actor (not the system);

- Collaboration task - a task carried out by two or more actors (humans and non-humans) such as the system (one or more agents/devices), therapists, and/or doctors. The nature of the interaction is collaborative (e.g., a task could not be done without the explicit participation of each actor);

- Communication task - a task performed by two or more actors (e.g., system, therapists, and/or doctors), to exchange information;

- Coordination task - a task performed by two or more actors (e.g., system, therapists, and/or doctors), that proceed in a coordinated way [8].

To map the outcomes presented in this section on the technological contributions offered by the current state of the art, a systematic methodology for reviewing scientific literature has been adopted.

The next section provides a quick overview of the executed steps, explicating the research questions that have been investigated.

\section{Systematic literature review: the methodology}

To provide a comprehensive overview of the current telerehabilitation systems, a systematic process has been adopted to retrieve, select, and analyze relevant literature.

\footnotetext{
${ }^{1}$ First phase after a surgical intervention on the knee. It is considered as over when the patient is able to passively perform a $90^{\circ}$ extension.
} 
This paper adheres to the procedure adopted and adapted by [1] and [24]. Therefore, this section focuses only on relevant methodological details. Rigorous and reproducible ${ }^{2}$ the methodology is composed of three stages (see Fig. 1).

Planning the review defines steps and constraints. In such a phase a generic free-form question (GFFQ) is broken-down in structured research questions (SRQs). Performing the review consists of: (i) collection and selection of relevant papers, (ii) elaboration of the selected papers, and (iii) feature extraction. Finally, the Document Review involves data analysis and reporting activities related to scientific dissemination.

\subsection{Research questions}

Concerning the requirements and the analysis provided in Section 2, to investigate how current technological solutions support telerehabilitation practices, the following main question arises:

- What type of system and support currently cope with telerehabilitation practices?

The GFFQ is decomposed in SRQs according to Goal-QuestionMetric (GQM) [25,26].

In relation with $\mathrm{T} 1$ and $\mathrm{T} 2$ (see Section 2), to understand the employment and to which extent the telerehabilitation systems target the real-world practices highlighted by the focus group, SRQ1 is defined:

- Concerning current telerehabilitation systems, what are the activities, scenarios, and designers and recipients involved?

In relation with $\mathrm{T} 3$ and $\mathrm{T} 5$ (see Section 2), to explore the most successful technologies employed in telerehabilitation, SRQ2 arises:

- What are the technologies primarily characterizing telerehabilitation systems?

Finally, to define the role played by MAS concerning T1-T5 (see Section 2) in telerehabilitation scenarios, SRQ4 is posed:

- What type of solutions, activities, characteristics, and features are offered by agent-based telerehabilitation systems?

\subsection{Searched channels and keywords}

We investigated the following peer-reviewed collections of papers: ieeeXplore, ${ }^{3}$ Sciencedirect, ${ }^{4}$ ACM Digital Library, ${ }^{5}$ Pubmed, ${ }^{6}$ and Citeseerx. ${ }^{7}$ We put additional effort to verify non-peer-reviewed literature, such arXiv e-Print, ${ }^{8}$ while ensuring sufficient coverage of the topic under study.

To perform a more accurate semi-automatic research, some keywords have been contextualized. Based on the reviewers' rooted backgrounds on MAS and assistive, and rehabilitation domains, the defined keywords follow: telerehabilitation, treatment at home, rehabilitation + telemedicine, telerehabilitation + MAS, telerehabilitation + multiagent system, telerehabilitation + agent-based systems, treatment at home + MAS, treatment at home + multi-agent systems, treatment at

\footnotetext{
${ }^{2}$ Primary studies selected and elaborated in January 2017 and updated in February 2018.

${ }^{3}$ http://ieeexplore.ieee.org/Xplore/home.jsp.

${ }^{4}$ http://www.sciencedirect.com/.

${ }^{5}$ http://dl.acm.org/.

${ }^{6}$ http://www.ncbi.nlm.nih.gov/pubmed.

${ }^{7}$ http://citeseerx.ist.psu.edu/index.

8 https://arxiv.org/.
}

home + agent-based systems, rehabilitation + telemedicine + MAS, rehabilitation + telemedicine + multi-agent systems, rehabilitation + telemedicine + agent-based systems. For each query, the papers crawlers produced lists of articles ordered by pertinence. The criteria used to stop paper collection is the same adopted by [1]: For each query, the articles collection has been stopped after a sequence of ten titles completely incoherent with the query performed appeared in results list. By "incoherence" it is meant that according to the reviewers' subjective view there was no adherence between the query performed and the title/abstract of the considered study.

\subsection{Inclusion criteria definition}

The initial collection counted 120 papers. A further coarse-grained examination, processing the compliance of the selected abstracts with the following inclusion criteria, reduced them to 26 .

(A) Context: The primary studies should define their contributions in the context of telerehabilitation/rehabilitation at home;

(B) Purpose: The purpose of the primary studies has to concern technological solutions that support rehabilitation-at-home practices;

(C) Practical foundation: The primary studies should provide at least one element from the following set: [practical design and implementation, tests, critical analysis, critical evaluations, and discussion].

\section{Results from the review}

This section presents the outcomes obtained by performing the methodology presented in Section 3. The structure of this section is framed according to the research questions presented above.

\section{1. $S R Q 1$}

Different approaches have been proposed according to patient conditions and medical requirements (e.g., fully outpatient, distant approach, or at-home) [27]. However, telerehabilitation solutions primarily target the older adult and patients from rural areas unable to reach medical centers [28]. Moreover, even in countries with excellent and capillary healthcare systems, telerehabilitation systems are firmly required. According to Carignan et al. [29], the main types of telerehabilitation interactions are:

(i) unilateral: patient and therapy are examined with a time-delay;

(ii) interactive bilateral: patient and therapist interact with each other through a virtual environment (e.g., video, virtual, and augmented reality) but without a direct force-feedback in either direction;

(iii) cooperative bilateral: therapist and patient interact directly with each other, still remotely, but with video, force, and kinesthetic feedback.

According to Mikolajewska et al. [30], the medical figures involved with the engineers in the development of telerehabilitation solutions are physician, physical therapists, occupational therapists.

Due to the lack of adequate studies concerning the patients, the interpretation of particular groups is restricted [31].

However, all of them are subject to a recovery period that usually lasts about six to eight weeks. It can follow an acute trauma (e.g., fall of a fragile older adult) or surgical intervention (e.g., joint replacement). This is the most critical period for patients who are fortunately still not chronic. Nevertheless, tailored solutions to relieve pain and maintain or slowly recover physical and/or mental capabilities have been developed by the scientific community. Indeed, telerehabilitation targets not only the physically impaired [32], but also cognitively impaired patients $[33,34]$ who are an average 76 years old (56-91) [31]. The most provided therapies are occupational, physical/motor-function, and 


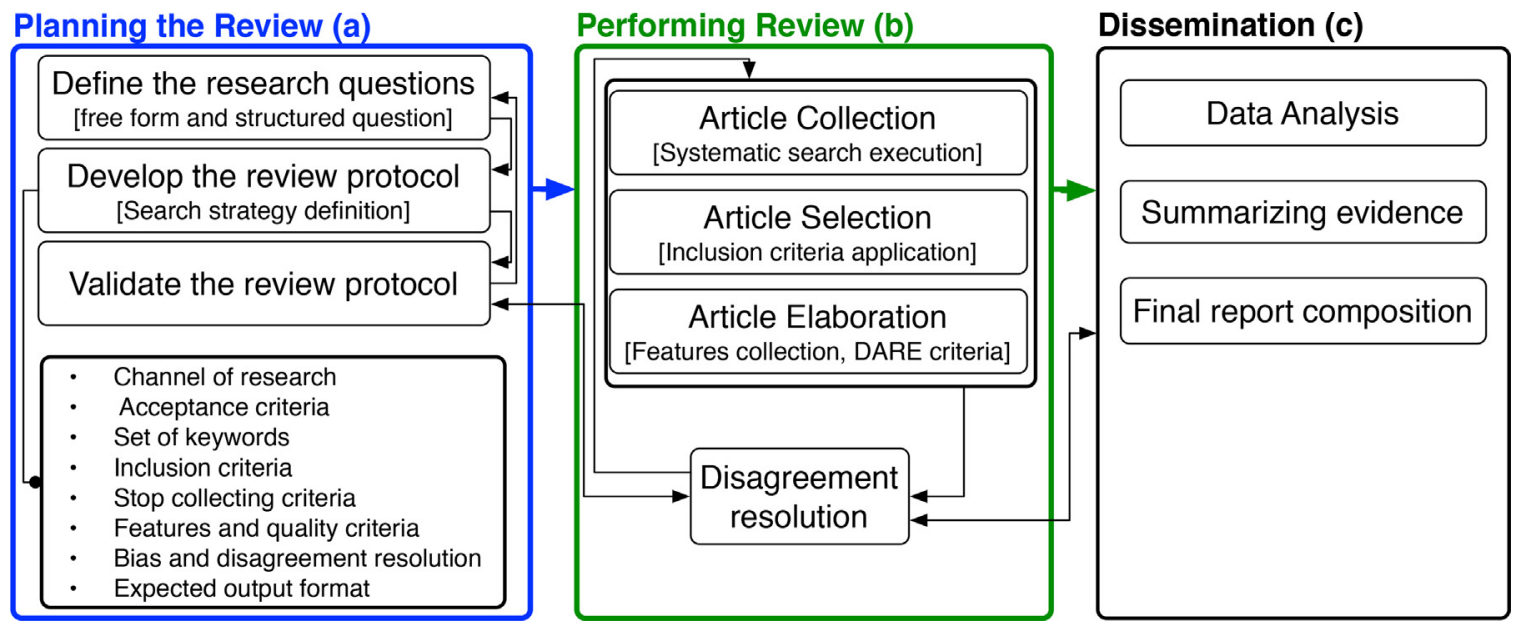

Fig. 1. Review methodology structure according to [1] and [25].

cognitive/neurological [13,30,35].

Depending on focus and point of delivery, telerehabilitation systems can cope with four main activities (i) training, (ii) counselling, (iii) monitoring, and (iv) assessment.

Haily et al. [4] counted twelve clinical categories supported by telerehabilitation systems, such as cardiology, neurology, cancer-related, speech disorders, urology, rheumatology, pulmonary, chronic pain, orthopaedic, morbidity, child obesity, age-related co-mobility.

Finally, most of the rehabilitation practices presented in Section 2 coping with technological artifacts can be turned in unassisted sessions. This entails in a number of recorded advantages such as (i) fastening the follow-up, (ii) enhancing the healing process, (iii) shortening the hospitalization, (iv) lowering the costs for both patients and health structures, (v) enabling continuous monitoring, (vi) providing equitable access to rehabilitation services, and finally (vii) supporting the technological advancement in telemedicine $[2,30]$.

\subsection{SRQ2}

The broad range of available technologies enabled the development of various techniques and approaches.

The main category of applications they have generated are based on:

- video analysis - involves stereoscopic cameras and image processing algorithms;

- wearable technology - focuses on embedded devices and inertial sensors supported by kinematic algorithms;

- robotics - focuses on in monitoring and motivation involving humanoids and basic robots;

- distributed sensing - involves monitoring and reasoning by exploiting environmental sensors;

- gamification - involves coaching techniques and persuasive technologies.

Despite the considerable availability of extremely precise and complex solutions, telerehabilitation systems have to face user acceptability.

The amount of similar proposed solutions suggests that the requirements set from physiotherapists and patients have not yet been entirely met. Factors such as setup, costs, maintenance, safety, easy usage, minimal set of options and functionalities, and effectiveness primarily impact on general acceptance and refusal [31].

\subsection{Video-based systems}

Nowadays, the demand for systems supporting both cognitive and motor stroke is quickly evolving. An application employing video-based technologies (e.g., virtual reality and video-elaborations) has been integrated into traditional rehabilitation practices, showing promising results.

Iarlori et al. [36] proposed a computer-vision based system applied to patients affected by Alzheimer's disease. The diagnosis of the illness's stage is performed by monitoring older adult in their private environment, and analyzing personal daily-care activities. Observing the actions listed in the Direct Assessment of Functional Status (DAFS) index and detecting performance anomalies helps to define the dementia stage. The authors analyzed teeth brushing and hair grooming using a Microsoft Kinect to collect data about the actions observed, and tracking the user's gestures. Thus, the patient can receive immediate support when incorrect or incoherent behaviors are detected.

Camirao et al. [37] proposed a first step investigating how VR could be employed in addressing the particular needs of this specific class of patients. Their study tried to assess the recovery of a cognitive-motor VR training with customized tasks and positive stimuli, compared to time-match conventional rehabilitation in the subacute phase of stroke. In such a context, a VR system (named Reh@Task) training attention, memory, and movements has been developed. Unfortunately, no explicit evidence showing relevant impact, with respect to standard rehabilitation methods, have been identified yet, particularly for patients with major cognitive impairment. Furthermore, the knowledge of how given stimuli in a certain virtual environment can affect the task performance and the overall patient recovery.

Lastly, Oliver et al. [38] developed a system tailored on cognitive and motor rehabilitation targeting aging individuals. It presents a strategic and extensible Rule Authoring component, that, considering the broad variety of cognitive injuries, offers a considerable number of possible exercises to treat them. The system primarily focus on Pair Association, Multiple Association, and Categorisation.

\subsection{Wearable-based systems}

Among all of the above mentioned approaches, wearable technology provided the most relevant information and is considered as the potential leader of further improvements in both preventative and rehabilitation approaches. On the other hand, camera-based applications still generate a number of concerns. A study targeting patients in an older adult-care facility revealed that $93 \%$ of the patients accepted body-worn sensor systems, defining them as non-invasive with little to no impact on normal daily activities [39]. 
Bergmann et al. [31] reported a surprisingly high consideration about the aesthetic of wearable sensors, as the patients were reluctant to be "stigmatized". Regarding the physiotherapists, major concerns arose for a restricted recording time due to limited storage capacity, wearability, and reliable real-time feedback.

According to Smith et al. [40], the current wearable devices successfully employed in telerehabilitation can be classified into 3 categories:

- Microsensors - capture health information by using small, intelligent, and low-energy active devices;

- Wrist devices - monitor health information by using combined sensors, a display, and wireless transmission in a single solution, which is very convenient for common physical activities;

- Smart clothes - capture information by using thin and flexible health sensors, compatible with textiles or made using textile technologies with specific properties (mechanical, electrical and optical).

Cesarini et al. [41] provided a highly customizable solution for supporting therapists and patients from the pre-surgical to the rehabilitation phase. Furthermore, they presented a particular implementation of a framework, involving two wearable inertial sensors and a tablet which can precisely monitor the angular position and velocity of the knee joint. Physicians and therapists can define specific exercises and related requirements (e.g., the number of repetitions, the number of steps and angular extension) characterizing the therapy. The system guides the patient during the execution of the exercises, providing a real-time visual feedback on the tablet, and evaluating the obtained performance at the end of the session.

Another study preformed by them presents a real-time feedback system of aquatic-space actions (e.g., performed by swimmers or rehabilitating patients) in the form of a functional sound, exploiting the so-called sonification procedure [42]. Such a system is composed of pressure sensors placed on the palmar and dorsal sides of the swimmer's hands, with a water-proof embedded system placed on the back of the swimmer. The pressure signals produced by the swimmer motion are processed by the embedded system and provided in real-time to both the swimmer and trainer/therapist. Furthermore, such a system can also be exploited in the context of rehabilitation activities and has already been presented in a specialized conference on aquatic therapy [43]. Therapists have widely accepted it as a promising tool for training and recovery of motor and coordination functions.

\subsection{Robotic-based systems}

Similar solutions involving robotic devices in the automation of rehabilitation procedure have been considered helpful in reducing training and rehabilitative sessions of both upper and lower extremities (well-known limitation of conventional methods) [20]. Indeed, taskoriented repetitive movements have a direct positive effect on improving muscle strength and movement in patients with neurological injuries. An automated robotic solution can acquire a higher number of exercise repetition compared to conventional approaches [44]. Eriksson et al. [45] realized an autonomous assistive mobile robot that provided monitoring, encouragement, and reminders to aid rehabilitating stroke patients. Navigating autonomously, it monitors the activity of the extremity in rehabilitation, reminding the patient to follow the program in the case of miss-behavior. Their experiments involved post-stroke patients. The proposed approach achieved positive responses about the increasingly active and animated robot behavior. The control system used is behavior-based which were characterized as pre- and postcondition to provide proper real-time feedback.

\subsection{Gamification-based systems}

Jacobs et al. [46] implemented a serious game to support arm-hand rehabilitation for stroke survivors. The main objective was to make the training effective and enjoyable. Exploiting task-oriented training principles, this game requires to manipulate every-day objects, dynamically adapting its difficulty based on patient performance. Both the physical and cognitive capabilities of two stroke patients were evaluated by the authors over a week.

Simmons et al. [47] studied a population of veterans with motor impairments diagnosed with acquired brain injuries. They examined the vet upper-limb motor function (manual muscle, goniometric range of motion, and dynamometer assessments) and executive functioning (testing cognitive functioning) using Exercise Games called PreMotor (PEGs). The evaluation of the conditions pre- and post-intervention showed that the participants demonstrated clinically relevant improvement regarding shoulder, elbow, and wrist strength. Thus, another success of the computer-based simulation driven intervention can be recorded, potentially leading towards lower demands of therapy with medical personnel. However, further research is needed to define which technologies better suit a given set of intervention.

From a technical and technological point of view, telerehabilitation systems are complex solutions which have to face context-rich scenarios, uncertainty, handle distributed sources of information, and operate in highly dynamic environments with mutual interdependencies and sophisticated distributed controls.

Although classic approaches have been shown as potentially effective, they lack in crucial features such as compatibility, collaboration, coordination, and communication [8].

Indeed, Miranda et al. [48] refer to common incompatibility problems such as data formats (e.g., storing format of 3D images) and different communication protocols. Such systems are either subject to an inevitable abandon, or require integrative upgrades (often unfeasible or require a worthless/unaffordable effort).

Therefore, studies such as Bergenti et al. [49] consider multi-agent systems (MAS) a suitable "technology" to realize such applications. Section 4.7 presents the most relevant agent-based telerehabilitation systems.

\subsection{SRQ3}

It is worth to recall that MAS are composed of several agents able to interact with each-other (e.g., their neighbors) for resource allocation, computational and decision-making tasks. The agents can share their information using the network interfaces to concur, enabling them to reach a common or private goal (e.g., consensus, synchronization, monitoring of health parameters [50] and surveillance [20]). Primarily due to these characteristics, MAS have been adopted in rehabilitation solutions attempting to cope with physical and cognitive phenomenons, and providing specialized models and tools.

\subsection{Physical rehabilitation}

Roda et al. [51] treated older adult motor impairment employing specific devices to control patient movement. Exploiting techniques typical of Ambient Intelligence (AmI), they proposed a context-aware system integrating diverse devices. Thus, MAS can react accordingly to the context, supporting physiotherapists in adapting and extrapolating from already existing therapies precisely tailored to patient need. Using a Microsoft Kinect, all the motor tasks performed by a patient during the rehabilitation are under control. Moreover, by employing thirdparty sensors, they were able to obtain oxygen level, posture, gesture, stress, BPM, and mood. Combining such values, pain or fatigue could also be detected. Physiotherapists expressed vague rules. For example providing a natural way to express how transitions should be made by using linguistic values rather than numerical values. A specific agent equipped with an inference engine elaborates such data while respecting isolation and privacy requirements.

Performing cardiac rehabilitation during the second (sub-acute) and 
third (intensive outpatient therapy) phase, a large amount of cardiac data (complex and arguably) has to be analyzed in a short period of time. The system proposed by Mesa et al. [52] provides support in data analysis, event classification, and visualization. Such a MAS has been involved in rehabilitative tests such as (i) walking on a treadmill at different speeds and inclinations; (ii) cycling on a stationary bike at different speeds; (iii) upper body workout; and (iv) lower body workout.

Data and context awareness is considered paramount to establish actual collaboration while interacting with remote participants. Dealing with rehabilitation systems magnifies this challenge. Hence, for both cognitive and physical rehabilitating users, the information awareness is a crucial element to provide patients with a rehabilitation path as tailored as possible [53].

In the context of Upper Limb Rehabilitation (ULR), Rodriguez et al. [8] proposed an agent-based system to customize exercises to assist different patients providing a bespoke ULR. A noteable peculiarity of such a system is the context-awareness, which enables run-time adaptability. Hence, the system "performs" three abstract concurrent tasks: (i) while the patient is executing the exercise for the upper limb, the movements are recorded and monitored; (ii) analysis of specific patient parameters (e.g., BPM, skin conductance), an agent is in charge of defining the level of stress/fatigue; (iii) the agent behaving as a "virtual therapist" adapts ULR's parameters such as number of repetitions and target area limits according to the current level of stress.

Felisberto et al. [54] developed a MAS that recognizes human movements, identifies human postures, and detects harmful activities in order to prevent risk situations (e.g., sudden diseases and falls). The authors exploited wireless sensor nodes and energy harvesting technologies to realize a wireless body area network (WBAN). On top of that, an intelligent agent constantly analyzes possible profiles variations, aiming at identifying physical and posture deterioration causing accidents.

Robotic manipulators have also been employed in agent-based solutions. Trainee learning phases may be supported by formalizing and enhancing the precision and the input to be understood [20]. Relevant contributions have been provided to the interaction between therapist, trainee, and patient.

In telerehabilitation scenarios, drugs assumption correlated to a highly dynamic environment can be a recurrent situation. Mutingi et al. [12] presented an agent-based decision-making solution for drugs delivering. The bio-physiological signals the authors took into account are blood-pressure, BPM, and respiration. Elaborating the combination of such parameters and drug therapy, the output may provide important indications about patient and pathology evolution to medical staff. Other benefits provided by this solution are staff workload reduction, increased resource availability, facilitation of patient requirement comprehension, and data collection.

\subsection{Cognitive rehabilitation}

In the scenario of cognitive rehabilitation, Abreu et al. [34] proposed a set of 3D games to rehabilitate neuropsychiatric disorders. They proposed an automatic agent-based control to facilitate the management of the software processes while the patient is playing.

Known as "the older adult silent epidemic", the Acquired Brain Injury (ABI) requires rehabilitation practices such as visuospatial, memory, functional communication, language, attention, and comprehension training [55]. Roda et al. [23] designed a MAS to (i) support the execution of the above-mentioned ABI related therapies, (ii) monitor and finally evaluate the performed activities and patient state (e.g., stress, emotional state, BPM, and oxygen level).

Smith et al. [40] proposed another agent-based solution for functional rehabilitation involving gamification. In an environment away from rehabilitation centers, such a solution promotes a continuous, fun, and stimulating rehabilitation. Such "games" have to carefully consider a higher number of variables (e.g., incorporating expertise and motivational capacities of rehabilitation practitioners). Thus, they result in being more complex than the ones offered by off the shelf, which are typically too physically and cognitively challenging for rehabilitation patients. Information about patient compliance and progress are collected and made available to the healthcare specialists for further analysis and considerations. Moreover, the gamification technique has been exploited seeking for an enhancement of the engagement, while performing monitoring and promoting smart learning mechanisms [56].

\subsection{Other proposed solutions}

Providing a platform for interactive learning, Su et al. [57] developed an ontology defining vocabulary, entities and their relationships in rehabilitation medicine. Exploiting an inference engine, existing data can reveal new knowledge having an "asserted model" as input and "inferred model" as output. Another example of agent-based reasoning is presented in [58]. The authors faced two main challenges: (i) scalability - by distributing the reasoning on mobile devices, and (ii) penalization by supporting medical staff with a graphical application simplifying the definition of temporal patterns of physiological values. Liao et al. [59] addressed reliability and security of an agent-based platform for telemonitoring.

Finally, Lai et al. [60] proposed a study involving a community scenario rather than the conventional single patient scenario. The authors evaluated the use of rehabilitation techniques for the post- or chronic-stroke survivors involving video-conferencing solutions. In conclusion, the authors praised efficacy, feasibility, and acceptability of telerehabilitation in community-dwelling stroke clients, recording improvements in both physical and psycho-social wellbeing.

\section{MAS for telerehabilitation: discussion}

According to the focus group (see Section 2.2), the major need in rehabilitation practices is to monitor a broad range of kinematic patient joint movement. Such an operation requires in-loco measurements, particularly exploiting wearable sensors which can guarantee a higher precision in terms of position over the time.

Although incorporating new technologies into rehabilitation and clinical service delivery achieved a high user satisfaction [61], this was unevenly higher for patients than therapists [11].

Promised advantages can naturally bring alongside them several drawbacks. For example, as deliberation time was extended, observation of physiological parameters in several solutions was neglected but was still delegated to the operator. Furthermore, fatigue, pain, and overall physical state are still not easy to quantify and analyze, by both a physiotherapist or a simple embedded system. Finally, technologically and technically heterogeneous systems introduce a considerable number of limitations.

Thus, a critical analysis can be formalized as follows:

On the one hand, embedded systems (no-MAS) can read and perceive in-loco both vital and kinematic parameters mainly related with step and task execution (see Section 2), but in the case a further analysis is required, heterogeneous and proprietary (so closed) solutions have to be involved. On the other hand, agent-based systems can easily provide sophisticated, extensible, and scalable analysis, supporting therapies and activities (see Section 2). However, being not possible yet to deploy MAS on embedded/wearable devices, adHoc solutions have to be employed, thus hindering and reducing the benefits/strengths characteristic of MAS.

\subsection{Strength}

Different patients present completely different scenarios. Expert 
agent-based systems are particularly good at modeling real-world and social systems where problems are solved in a concurrent and cooperative way, without the need of reaching optimal solutions [51]. Therefore, MAS are potentially able to dynamically relate and contextualize vital parameters and rehabilitative practices.

The adoption of MAS is crucial for activities such as decision making. Remote diagnosis, treatment adaption, treatment planning, identification of potentially dangerous situations, knowledge representation and manipulation are the key features common to most rehabilitative scenarios and pervasive cares [51]. Hence, such transparent and intelligent mechanisms might embed in a single comprehensive solution a broad variety of services, only limited by the "number" and "expertise/capabilities" of the involved agents.

Combining all those features, emergency mechanisms could even make decisions in the absence of a human decision maker [12]. Moreover, the response time (e.g., in terms of data analysis) would be significantly reduced, especially if considering possible accuracy and consistency. However, providing time guarantees (dealing with strict timing constraints) is still a weakness of current MAS. The next section presents some limitations and their consequences.

\subsection{Limitations}

The multi-agent paradigm has been utilized to solve several types of problems. However, current MAS remain unpredictable and unable to respect strict timing constraints. Hence, they introduce drawbacks related to theoretical models, single applications and the agent technology itself.

A prominent example is one of the major problems for solutions involving robotic operators [20]: the need to increase units.

Regarding the multi-agency, the common disadvantages already claimed in the scientific literature [12] are:

- complexity - the higher level of the systems' complexity requires more expertise and training;

- human-relationship - dealing with "virtual entities", patients are concerned about the risk of being disconnected/abandoned by the therapist;

- security - named as "possible technology perversion", refers to ethical and security concerns.

However, by analyzing the contributions collected in Section 4.7, the above list can be extended to include the disadvantages of technical and technological limitations/problems of MAS which inhibit their adoption and limit their benefits (see Table 1).

Fig. 2 schematically illustrates the general composition of agentbased rehabilitation systems. MAS only appear in the "higher levels" of every system. For example, in [52] and [54] the only elements exploiting agents are the data handler, visualizer, and alert manager. In other contributions, such as [51], references or details about how the agents get information from wearable sensors or embedded devices are often missing or omitted.

In particular, concerning the attempt of coupling MAS and ad-Hoc embedded systems, Calvaresi et al. [62] proposed a study involving a mobile robot composed of a Pandaboard, a Discovery STM32, two DC motors and a camera. The MAS (running on the Pandaboard) has been realized using JADE [63] and it was in charge of performing all the dynamics related to vision and intelligent planning. The robot's motion was managed by an ad-Hoc non-MAS system running on the Discovery board. Such an element and its functionalities have been wrapped in one of the agents running on the main board implementing a custom communication protocol. Such a best-effort solution identified by the authors has been "forced" by the impossibility of running MAS and JVM on the STM32, which is due to its limited resources and the incompatibility of JVM (and so JADE) with Erika RTOS [64].

Investigating the difficulties and barriers encountered in such experience, the authors of [62] frame the limitations arising when MAS are required to deal with embedded systems. A formalization of such limitation is given in Table 1.

To employ MAS in safety-critical and cyber-physical systems, overcoming the limitations listed above is mandatory. Nevertheless, some radical changes are required. The next section presents them, giving particular emphasis to those strictly required by the telerehabilitation domain.

\subsection{Open challenges}

The main challenge concerning MAS in telerehabilitation scenarios is the following:

"To bring the agent capabilities and proprieties at the sensing level".

Moving from the current rehabilitation MAS (Fig. 2), which only partially exploits agent capabilities, towards a solution that employs agents at any level, would represent a crucial step for the multi-agent community involving the resolution of all the limitations presented in Section 5.2.

For example, Fig. 3 shows a possible agent-based wearable system for knee rehabilitation endorsing such a radically new challenge. In particular, deploying agents directly on distributed sensors eliminates the need for ad-hoc wrappers, workarounds, and overly tailored patches. Such a design embodies the need for overcoming the limitations listed in Table 1.

Sensing in rehabilitation has to be performed at different levels, and thus, it requires diverse sensors and tasks matching specific situations. Delegating the sensing directly to a set of agents spread on the wearable sensors can produce concrete benefits.

However, sensing implies understanding and correlating the exact sensor position at the exact time for the entire execution of the exercises. Employing intelligent agents in such a process mainly implies the strict consideration of constraints such as (i) scarcity of resources, (ii) new communication means, and (iii) timing constraints.

\subsection{Scarcity of resources}

Most wearable devices have limited resources (e.g. memory and computational capability) to have a contained impact on the battery lifetime.

Moreover, they are also subject to dimensional restrictions due to wearability and intrusivity factors. Thus, most of the conventional multi-agent frameworks cannot be deployed on such devices. Intervention to lighten agents and communication protocols are envisioned to finally remove the barriers from the employment of MAS in

Table 1

MAS for CPS: limitations.

\begin{tabular}{ll}
\hline $\mathrm{n}$. & Description \\
\hline 1 & The need for custom communication protocols unable to respect the FIPA standard [65]. \\
2 & The impossibility of running agent programmed with interpreted languages (e.g., Java) on embedded devices with constrained resources. \\
3 & The difficulty of guaranteeing the respect of strict timing constraints. \\
4 & The current impossibility of running agent-based systems on real-time operating systems (e.g., Erika Enterprise [64]).
\end{tabular}




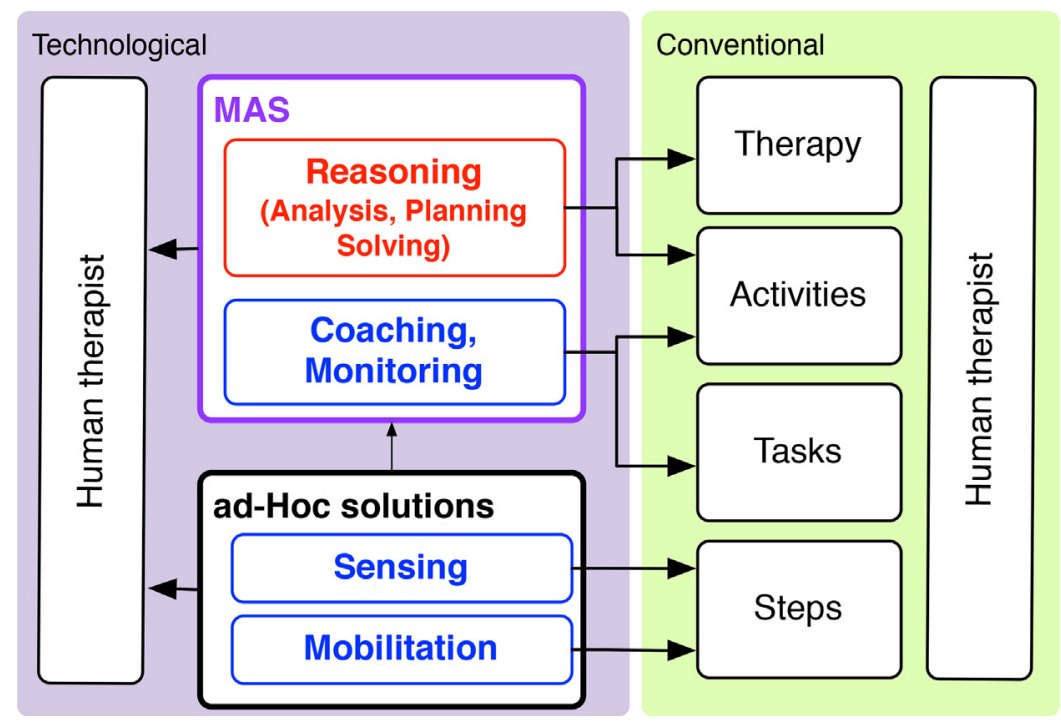

Fig. 2. Rehabilitation MAS structure.

embedded devices.

\subsection{Communication means}

Wearable devices for telerehabilitation are usually characterized by low-energy consumption communication means: Bluetooth low-energy (BLE) [66] and Zigbee [67]. Standard communication protocols (e.g., FIPA ACL [65]) need to be updated to suite such new channels of communication. Indeed, such protocols, broadly used in Ethernet or WiFi connection, need to take a new (and more constrictive) set of constraints into consideration. Finally, by collaborating to ensure the respect of strict timing constraints, the new communication protocols must take into account boundaries and theories typical of distributed real-time applications.

This work considers the Timing constraints a crucial challenge of primary importance for safety-critical, therefore telerehabilitation, systems. For example, jeopardized values from the computation of the kinematic information within a sensor node, and the communication among them due to a lack of timing reliability, may cause dramatic consequences to the patient. The next section focuses on how current
MAS face the problem of meeting timing constraints (if possible) providing the fundamental notions to overcome the current limitations.

\section{Timing constraints}

The major medical outcome emerging in Section 2.2 is the need for systems with the ability to provide precise information (in time and space), and to reason on raw data, providing human-understandable aggregated data to coach both patients and doctors. Concerning the analysis of agent-based solutions provided in Section 5, the crucial lack of guarantee for the compliance with strict timing constraints, necessary to cope with medical requirements, became apparent [68]. Hence, current MAS, both in terms of theoretical models and in practical implementations, (platforms/frameworks), do not yet have mechanisms to deal with "strict timing constraints" [69]. The inner functionalities and interactions of current implementations do not provide the possibility of facing safety-critical scenarios. As a consequence, a critical failure could lead to injuries, environmental damage, and even financial losses. In the case of telerehabilitation, a delayed feedback might increase the risk of a serious injury (e.g., in a coaching system, an overrunning task

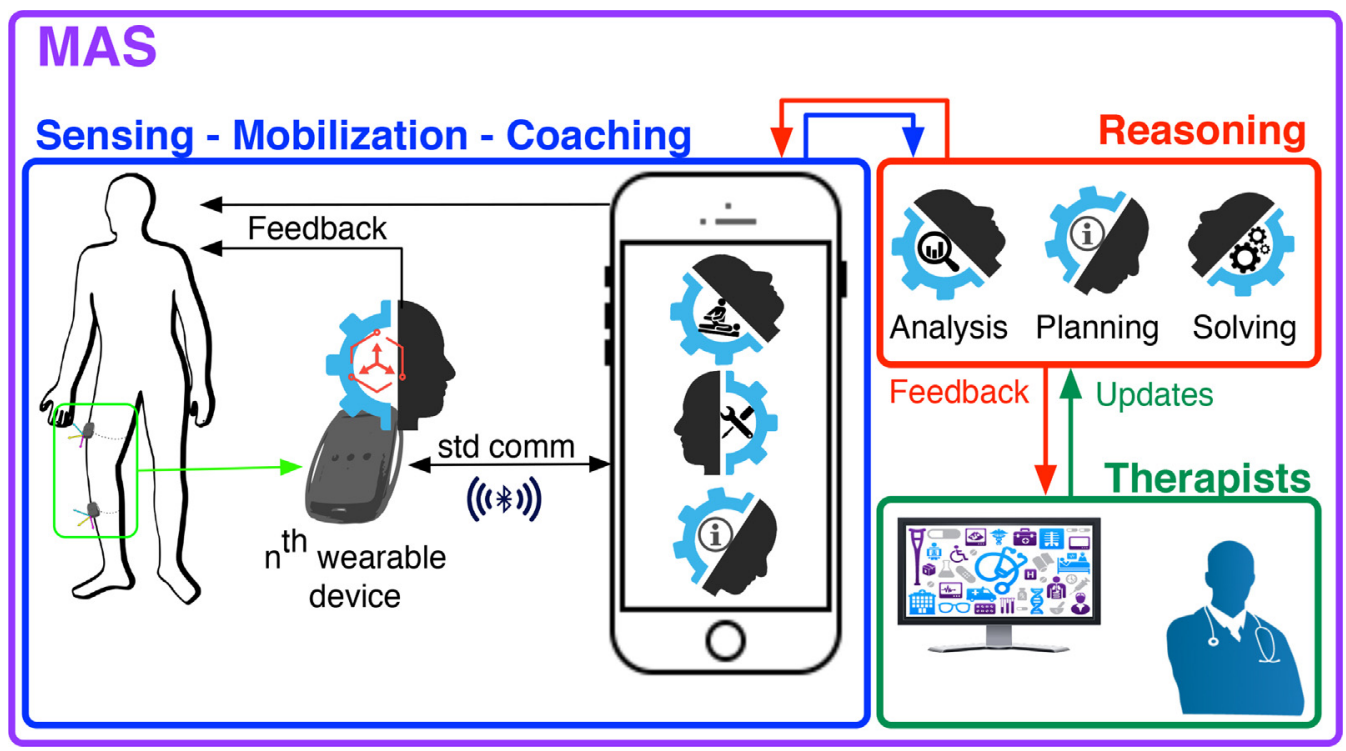

Fig. 3. Agent-based sensing: future challenge for telerehabilitation MAS. 
might require the patient to continue a movement over the limit of its safety range).

To prevent such risks, the agents involved in such systems have to guarantee the respect of strict timing constraints. Such guarantees are achievable only if, at the same time, all the MAS pillars (the local scheduler, scheduling the behaviors inside a given agent, the negotiation protocol, and communication protocols) consider specific factors [69] in their inner mechanisms. Hence, having a single mentioned element incapable of dealing with timing guarantees makes providing any guarantee on predictable behaviors impossible.

Concerning local schedulers, agent tasks are usually referred to as simple or complex behaviours. The majority of the existing MAS are powered by specific platforms supporting the development of agentbased systems. According to the state of the art, almost all those platforms (except two) have implemented at least one local scheduler. The first platform declaring the absence of any specific scheduler implementation, thus delegating its design and coding to the user, is NetLogo [70]. The only support provided to the developer is a default event-driven mechanism characterizing the platform which might be used to process the agent behaviors. The second platform is Cormas [71], which, differently from the previous one, if no custom/Ad-Hoc scheduler is provided, the behaviors are not executed (nothing in the system would happen). This allows the platform users to directly implement their version of a behavior scheduler, ensuring high flexibility. Hence, not only pure algorithms are admitted (e.g., heuristics such as round-robing (RR), random selection, less workload first, early starting time first), and the development of custom mixes of the above-mentioned algorithms is also encouraged [72]. MaDKit [73], RePast [74], and Swarm [75] implement the classic first-come-first-served (FCFS). GAMA [76] and MASON [77] implement a type of priority scheduler (e.g., SJF-like). Jason implements an RR applied to structured behaviors. JADE implements a non-preemptive RR [78]. The Jason and Jade implementations of RR result in being FCFS of intentions [79] in the first case, and of behaviors in the second, which are eventually treated like single entities.

Depending on the various behaviour characteristics (e.g., periodic or sporadic), several schedulers typical of real-time embedded systems can be employed. For example, depending on the need of fixed or $d y$ namic priority, schedulers such as Rate Monotonic (RM) [80] or Earliest Dead-line First (EDF) [18] can be considered to be employed. Both of them are based on the analysis of the worst-case scenario for the taskset under evaluation, thus able to guarantee a correct resource/task allocation and the respect of timing constraints. In the case of less predictable aperiodic behaviors (e.g., tasks that might generate overrun), the most suitable approach would employ a scheduler based on the concept of servers such as the Sporadic Server (SS), Total Bandwidth Server (TBS), and Constant Bandwidth Server (CBS) [18]. Thus, the maximum computation bandwidth of incoming requests can be bounded for each task or class of tasks, providing isolation among them and reducing the pessimism in the timing analysis.

Recalling that the task utilization factor is a fraction of processor time spent in the execution of single task, the processor utilization factor $\left(U_{p}\right)$ is the utilization of the entire task-set [18]. It is calculated as show in Eq. (1), where $C_{i}$ is the worst-case execution time and $T_{i}$ is the period of a ith task.

$U_{p}=\sum_{i=1}^{n} \frac{C_{i}}{T_{i}}$

It is worth to recall that the sensor nodes and many MAS rely on hardware characterized by single cores (physical or virtual). For this class of systems, if $U_{p}>1$, no scheduling algorithm can guarantee the correct execution of such a task-set (without missing any deadline). The task-set is defined not feasible. Vice-versa, several scheduling algorithms can guarantee the respect of all deadlines. Despite the optimality for RM [18], the fact that maximum $U_{p}$ for which it can ensure timing guarantees is quite low. Moreover, its performance dramatically depend on the task-set features. The lower upper bound of the processor utilization factor $\left(U_{l u b}\right)$ is the minimum of the utilization factor among all task sets that fully utilize the processor. In fact, any task set whose processor utilization factor is less than or equal to this bound, is schedulable by the algorithm. On the other hand, when $U_{\text {lub }}<U_{p} \leq 1.0$, the schedulability can only be achieved if the task periods are suitably related. The $U_{\text {lub }}$ of RM is shown in Eq. (2), and for $n \rightarrow \infty, \quad U_{\text {lub }}^{\mathrm{RM}} \rightarrow \ln 2$.

$U_{\text {lub }}^{\mathrm{RM}}=n\left(2^{1 / 2}-1\right)$

Considering EDF, its lower upper bound is set at $U_{\text {lub }}^{\mathrm{EDF}}=1$. This means that if the tasks-set is feasible, it is schedulable by EDF and might be schedulable by the other above-mentioned algorithms.

The CBS offers the same advantages of EDF (since they share the same mechanism) and can also deal with aperiodic requests. Moreover, it provides isolation mechanisms by proposing and efficiently implementing a bandwidth reservation strategy.

The CBS mechanism relies on the basic idea of introducing the concept of server, which is a periodic task whose purpose is to serve aperiodic requests as soon as possible. Its computational time (budget) is indicated with $Q_{s}$, its period is indicated with $P_{s}$, and the ratio $U_{s}=Q_{s} / P_{s}$ denotes its bandwidth.

When a new task enters the system (maintaining that the task-set is still schedulable), a suitable scheduling deadline is assigned (to bound its execution in the reserved bandwidth), followed by its insertion (accordingly to its deadline) in the EDF ready queue. If the job tries to execute more than expected, its deadline is postponed. Such a task is still eligible for being executed, but its priority is decreased minimizing its interference on the other tasks.

For those schedulers which make various use of the concept of server, the system utilization factor is the sum of the processor utilization factor (see Eq. (1)) and server utilization factor (see Eq. (3)). Thus, the final value is given by Eq. (4).

$U_{s}=\sum_{s=1}^{m} \frac{Q_{s}}{P_{s}}$

$U_{\text {sys }}=U_{p}+U_{s} \leq 1$

Note that, if a subset of tasks is handled by a single server, all the tasks in that subset will share the same budget/bandwidth, so there is no isolation among them. Nevertheless, all the other tasks in the system are protected against overruns occurring in any server.

Finally, the advantages directly guaranteed by the enrollment of the CBS as local scheduler are:

- High utilization with bounded response times.

- Respect of strict timing constraints (no deadline misses).

- Tractable acceptance test (executed during bid).

- Isolation among periodic and aperiodic tasks to avoid/minimize interference.

Agent interactions rely on the communication middleware, defining common (possibly standard) formats and semantics. FIPA [65] is the reference standard and is characterized by messages strictly adhering to the Agent Communication Language (ACL) standard, enabling several possible encoding of the actual contents. Once the message is composed, it is easily sent over IP. However, no mechanism to manage (i) network load and messages status (e.g., the impossibility of bounding congestions and delivering times), (ii) in/out message queues, and (iii) broadcasting (e.g., broadcasting simple sensors values still require a complex management) is provided. To overcome such limitations, a real-time publish-subscribe (RTPS) communication mechanism might be employed. Hence, systems such as the Data Distribution Service (DDS) [81] implement a version of RTPS to improve the predictability of transmission times, managing the quality of service for the 
Table 2

System's tasks/behavior list.

\begin{tabular}{llll}
\hline Task & Behavior & Task & Behavior \\
\hline$\tau_{1}$ & Reading messages & $\tau_{2}$ & Writing messages \\
$\tau_{3}$ & Computing inertial information & $\tau_{4}$ & Displaying graphically \\
$\tau_{5}$ & Starts the rehabilitation session & The elaborated inertial information
\end{tabular}

transmitted packages and the scalability of the system.

The need for a distributed coordination of tasks and resources among multiple problem solvers (nodes/agents) generated many different contributions over the years. Although flexibility is always guaranteed, none of the current negotiation protocols, in charge of ruling such distribution, can ensure any temporal bound or the possibility of a positive conclusion of a specific transaction. Although such fascinating and sophisticated mechanisms are suitable for dynamic systems, the negotiation process is still unpredictable. Thus, resulting in being unemployable in safety-critical or strictly time-dependent scenarios. Therefore, negotiation mechanisms should envision a strict connection with the other MAS components (agent internal scheduler and communication middleware). An example is the acceptance of a task execution demanded in the negotiation phase which would impact on the contractor's task-set (agent proposing for the task execution). In such a way, task-related parameters (e.g., worst-case execution time, inter-arrival time, and activation time) and agent-related parameters (e.g., communication delay and utilization factor) must be mandatorily taken into consideration if aiming at negotiating under real-time constraints. Thus, both tasks already accepted and running on the agent (contractor) and tasks under bids will have ensured the promised response time undertaken during the negotiations.

Facing these new challenges requires a substantial intervention within the inner mechanisms of traditional MAS. Nevertheless, the operating principles and basic protocols will still be respected, thus enabling interactions and collaborations with agents implemented according to the current policies. This is demonstrated by MAS performing long-term reasoning and data handling, operating in non-safety critical scenarios, with the possibility to be implemented in the traditional way. Although incapable of guaranteeing the compliance with the newly presented constraints, such traditional agents can elaborate data provided by the real-time agents running on the wearable devices.

\section{Application of the proposed solution in a knee telerehabilitation therapy}

In the context of lower-limb telerehabilitation [2], this section models and analyzes a system based on the multi-agent approach.

It is worth to recall that AI and MAS have already brought valuable contributions to medicine and telemedicine application $[1,14,15]$. However, in the framework of medical practices demanding embedded and wearable systems $[2,82]$, enabling MAS to produce reliable and medically valid results is still an open challenge [69]. For example, to guarantee the correct computation of the knee angle with the accuracy demanded by the physician, measurements and analysis must ensure the complete absence of possibly jeopardized data (i.e., time-wise misaligned measurements can provide erroneous spatial information).

Supporting the relevance and the need for such systems, in the literature we can find similar applications attempting at dealing with similar practices $[2,35,41]$. However, no study/application has been able to employ agents on distributed sensors for medical purposes.

To serve telerehabilitation practices (e.g., single- and multi-joint telerehabilitation), the proposed solution extends the already existing advantages granted by MAS $[14,83]$, providing dynamical deployment and autonomous configuration of wearable inertial sensors. Moreover, it can ensure reliable (time- and space-wise) kinematic information of the rehabilitated joint.

The fundamental innovations of the presented agent-based design are a reservation based negotiation protocol relying and the CBS mechanism employed as local scheduler.

The objective of the system is to plot a graphical representation of the kinematic characterizing a knee during a telerehabilitation session on a tablet. The system is composed of three agents, possibly being proactive and/or reactive. In our system they are divided as follows:

- The similar agents $\mathcal{B}$ and $C$ are deployed on wearable sensors capable of computing and sharing inertial information (reactive agents).

- The agent $\mathcal{A}$ (proactive agent) runs on a tablet and is in charge of integrating and displaying the values received from $\mathcal{B}$ and $C$.

The behaviors/tasks running in the system are listed in Table 2:

For simplicity, in this example, the communication delays among the agents are assumed to be constant (i.e., $\left.\delta_{\mathcal{A}, \mathcal{B}}=\delta_{\mathcal{B}, \mathcal{A}}=\delta_{\mathcal{A}, C}=\ldots=\delta_{\text {comm }}\right)$. Such a value is included in the computation time of each communication task (i.e., $\tau_{1}$ or $\tau_{2}$ ).

The task-set of agent $\mathcal{A}\left(\Gamma^{A}\right)$ is composed of $\tau_{1}, \tau_{2}, \tau_{4}, \tau_{5}$. The tasksets of agents $\mathcal{B}\left(\Gamma^{B}\right)$ and $C\left(\Gamma^{C}\right)$ have the same composition which is $\tau_{1}$, $\tau_{2}, \tau_{3}$. The task computation time and period are specified in Table $3 \mathrm{a}$. The system dynamics are represented in Fig. 4(a).

Such tasks are at least characterized by computation time $\left(C_{i}\right)$ and absolute deadline $\left(D_{i}\right)$. Moreover, the parameter $\left(T_{i}\right)$ indicates the period

Table 3

Agents' task-sets.

\begin{tabular}{|c|c|c|c|c|}
\hline Agent & $\mathrm{t}$ & & C & $\mathrm{T}$ \\
\hline \multicolumn{5}{|c|}{ (a) Tasks parameters } \\
\hline $\mathcal{A}, \mathcal{B}, C$ & $\tau_{1}$ & & 1 & - \\
\hline $\mathcal{A}, \mathcal{B}, \mathcal{C}$ & $\tau_{2}$ & & 1 & - \\
\hline $\mathcal{B}, C$ & $\tau_{3}$ & & 6 & 20 \\
\hline $\mathcal{A}$ & $\tau_{4}$ & & 4 & 20 \\
\hline $\mathcal{A}$ & $\tau_{5}$ & & 1 & - \\
\hline Server & & Q & & $\mathrm{T}$ \\
\hline \multicolumn{5}{|c|}{ (b) Serves' parameters } \\
\hline$s_{1}$ & & 2 & & 20 \\
\hline$s_{2}$ & & 2 & & 20 \\
\hline$s_{5}$ & & 1 & & 20 \\
\hline
\end{tabular}




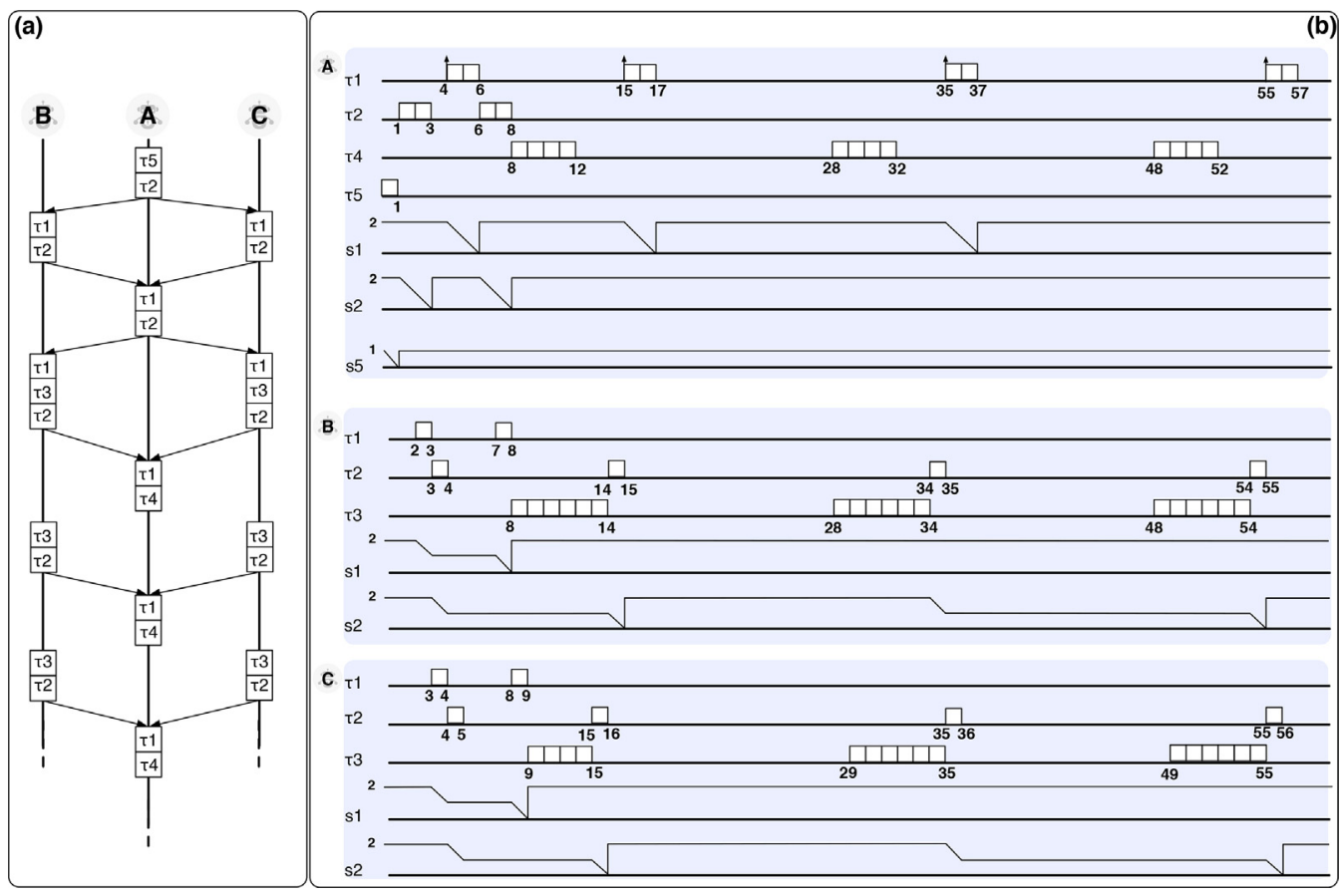

Fig. 4. System representation in: (a) AUML, (b) tasks scheduling.

Table 4

Events log of agent $\mathcal{A}$.

\begin{tabular}{|c|c|c|c|c|c|c|}
\hline Time (ms) & $\Gamma_{\mathcal{A}}$ & $U_{\mathcal{H}}$ & $\tau_{k}$ req. CPU & Action & Deadline $\left(d_{\tau_{k}}=r_{\tau_{k}}+T_{\tau_{k}}\right)$ & Exec \\
\hline$t=1$ & $\tau_{1}, \tau_{2},\left(\tau_{4} ?\right)$ & $0.2(0.4 ?)$ & $\tau_{2}$ & (writing to $\mathcal{B}$ ) & $d_{\tau_{2}}=1+20=21$ & {$[1,2]$} \\
\hline$t=4$ & $\tau_{1}, \tau_{2},\left(\tau_{4} ?\right)$ & $0.2(0.4 ?)$ & $\tau_{1}$ & (reading from $\mathcal{B}$ ) & $d_{\tau_{1}}=4+20=24$ & {$[4,5]$} \\
\hline$t=5$ & $\tau_{1}, \tau_{2},\left(\tau_{4} ?\right)$ & $0.2(0.4 ?)$ & $\tau_{1}$ & (reading from $C$ ) & $d_{\tau_{1}}=5+20=25$ & {$[5,6]$} \\
\hline$t=6$ & $\tau_{1}, \tau_{2}, \tau_{4}$ & 0.4 & $\tau_{2}$ & (writing to $\mathcal{B}$ ) & $d_{\tau_{2}}=6+20=26$ & {$[6,7]$} \\
\hline$t=7$ & $\tau_{1}, \tau_{2}, \tau_{4}$ & 0.4 & $\tau_{2}$ & (writing to $C$ ) & $d_{\tau_{2}}=7+20=27$ & {$[7,8]$} \\
\hline$t=16$ & $\tau_{1}, \tau_{2}, \tau_{4}$ & 0.4 & $\tau_{1}$ & (reading from $C$ ) & $d_{\tau_{1}}=16+20=36$ & {$[16,17]$} \\
\hline$t=28$ & $\tau_{1}, \tau_{2}, \tau_{4}$ & 0.4 & $\tau_{4}$ & (plotting values) & $d_{\tau_{4}}=28+20=48$ & {$[28,32]$} \\
\hline$t=35$ & $\tau_{1}, \tau_{2}, \tau_{4}$ & 0.4 & $\tau_{1}$ & (reading from $\mathcal{B}$ ) & $d_{\tau_{1}}=35+20=55$ & {$[35,36]$} \\
\hline$t=36$ & $\tau_{1}, \tau_{2}, \tau_{4}$ & 0.4 & $\tau_{1}$ & (reading from $C$ ) & $d_{\tau_{1}}=36+20=56$ & {$[36,37]$} \\
\hline$t=48$ & $\tau_{1}, \tau_{2}, \tau_{4}$ & 0.4 & $\tau_{4}$ & (plotting values) & $d_{\tau_{4}}=48+20=68$ & {$[48,49]$} \\
\hline
\end{tabular}

and so forth ....

Table 5

Events $\log$ of agent $\mathcal{B}$.

\begin{tabular}{|c|c|c|c|c|c|c|}
\hline Time (ms) & $\Gamma_{\mathcal{A}}$ & $U_{\mathcal{A}}$ & $\tau_{k}$ req. $\mathrm{CPU}$ & Action & Deadline $\left(d_{\tau_{k}}=r_{\tau_{k}}+T_{\tau_{k}}\right)$ & Exec \\
\hline$t=2$ & $\tau_{1}, \tau_{2}$ & 0.2 & $\tau_{1}$ & (reading from $\mathcal{A}$ ) & $d_{\tau_{1}}=2+20=22$ & {$[2,3]$} \\
\hline$t=3$ & $\tau_{1}, \tau_{2},\left(\tau_{3} ?\right)$ & $0.2(0.5 ?)$ & $\tau_{2}$ & (writing to $\mathcal{A}$ ) & $d_{\tau_{2}}=3+20=23$ & {$[1,2]$} \\
\hline$t=7$ & $\tau_{1}, \tau_{2},\left(\tau_{3} ?\right)$ & $0.2(0.5 ?)$ & $\tau_{1}$ & (reading from $\mathcal{A}$ ) & $d_{\tau_{1}}=7+20=27$ & {$[7,8]$} \\
\hline$t=8$ & $\tau_{1}, \tau_{2}, \tau_{3}$ & 0.5 & $\tau_{3}$ & (computing angular value) & $d_{\tau_{3}}=8+20=28$ & {$[8,14]$} \\
\hline$t=14$ & $\tau_{1}, \tau_{2}, \tau_{3}$ & 0.5 & $\tau_{2}$ & (writing to $\mathcal{A}$ ) & $d_{\tau_{2}}=14+20=34$ & {$[14,15]$} \\
\hline$t=28$ & $\tau_{1}, \tau_{2}, \tau_{3}$ & 0.5 & $\tau_{3}$ & (computing angular value) & $d_{\tau_{3}}=28+20=48$ & {$[28,34]$} \\
\hline$t=34$ & $\tau_{1}, \tau_{2}, \tau_{3}$ & 0.5 & $\tau_{2}$ & (writing to $\mathcal{A}$ ) & $d_{\tau_{2}}=34+20=54$ & {$[34,35]$} \\
\hline
\end{tabular}

and so forth ....

for the periodic tasks and the minimum-interarrival time for the sporadic tasks. Finally, for such a task-set, $T_{i}=D_{i}$ has been assumed.

It is worth to recall that this case-study employs assumptions characterizing the traditional schedulability analysis, which are:
- The instances of a periodic task $\tau_{i}$ are regularly activated at a constant rate. The interval $T_{i}$ between two consecutive activations is the period of the task.

- All instances of a periodic task $\tau_{i}$ have the same worst-case execution time $C_{i}$. 
Table 6

Events $\log$ of agent $C$.

\begin{tabular}{|c|c|c|c|c|c|c|}
\hline Time (ms) & $\Gamma_{\mathscr{A}}$ & $U_{\mathcal{A}}$ & $\tau_{k}$ req. CPU & Action & Deadline $\left(d_{\tau_{k}}=r_{\tau_{k}}+T_{\tau_{k}}\right)$ & Exec \\
\hline$t=3$ & $\tau_{1}, \tau_{2}$ & 0.2 & $\tau_{1}$ & $($ reading from $\mathcal{A})$ & $d_{\tau_{1}}=3+20=23$ & {$[3,4]$} \\
\hline$t=4$ & $\tau_{1}, \tau_{2},\left(\tau_{3} ?\right)$ & $0.2(0.5 ?)$ & $\tau_{2}$ & (writing to $\mathcal{A}$ ) & $d_{\tau_{2}}=4+20=24$ & {$[4,5]$} \\
\hline$t=8$ & $\tau_{1}, \tau_{2},\left(\tau_{3} ?\right)$ & $0.2(0.5 ?)$ & $\tau_{1}$ & (reading from $\mathcal{A}$ ) & $d_{\tau_{1}}=8+20=28$ & {$[8,9]$} \\
\hline$t=9$ & $\tau_{1}, \tau_{2}, \tau_{3}$ & 0.5 & $\tau_{3}$ & (computing angular value) & $d_{\tau_{3}}=9+20=29$ & {$[9,15]$} \\
\hline$t=15$ & $\tau_{1}, \tau_{2}, \tau_{3}$ & 0.5 & $\tau_{2}$ & (writing to $\mathcal{A}$ ) & $d_{\tau_{2}}=15+20=35$ & {$[15,16]$} \\
\hline$t=29$ & $\tau_{1}, \tau_{2}, \tau_{3}$ & 0.5 & $\tau_{3}$ & (computing angular value) & $d_{\tau_{3}}=29+20=49$ & {$[29,35]$} \\
\hline$t=35$ & $\tau_{1}, \tau_{2}, \tau_{3}$ & 0.5 & $\tau_{2}$ & (writing to $\mathcal{A}$ ) & $d_{\tau_{2}}=35+20=55$ & {$[35,36]$} \\
\hline
\end{tabular}

and so forth ....

- All instances of a periodic task $\tau_{i}$ have the same relative deadline $D_{i}$, which is equal to the period $T_{i}$.

- All tasks in all the $\Gamma^{*}$ are independent; which means that there are no precedence relations and no resource constraints.

For completion, the implicit assumption involved by $A 1, A 2, A 3$, and A4 are listed below:

- No task can suspend itself, for example on I/O operations.

- All tasks are released as soon as they arrive.

- All overheads in the kernel are assumed to be negligible.

\subsection{The role of the CBS}

As introduced in Section 6, the CBS can provide isolation among aperiodic and periodic tasks. In this case study, $\tau_{1}, \tau_{2}$, and $\tau_{5}$ are aperiodic, having different characteristics and scopes. Therefore, the common practice is to assign them to independent servers [18] (e.g., $\tau_{1} \rightarrow s_{1}, \tau_{2} \rightarrow s_{2}$, and $\tau_{5} \rightarrow s_{5}$ ), characterized as shown in Table $3 \mathrm{~b}$ where $P_{s}=T_{s}$ and $C_{s}=Q_{s}$.

When the system starts, at $t=0, \mathcal{A}$ has only scheduled $\tau_{1}, \tau_{2}, \tau_{5}$. Thus, according to Eq. (4) its utilization factor is $U=0.25$. At the same instant, according to the same formula, $\mathcal{B}$ and $C$ have $U=0.2$, since they only have $\tau_{1}$ and $\tau_{2}$ in the set task.

The execution of task $\tau_{5}$ (at $t=1$ ) generates in $\mathcal{A}$ the need for information produced by the execution of task $\tau_{3}$ from both $\mathcal{B}$ and $C$. If adding such a task to the analysis Eq. (4) is still respected and if the negotiation for on agents $\mathcal{B}$ and $C$ get accomplished, task $\tau_{4}$ is added to the task-set. Considering that $U_{\tau_{4}}=0.2$, we have $U^{\mathcal{H}}=0.4 \leq 1$, so the task-set of $\mathcal{A}$ is still schedulable. The contribution in terms of $U_{i}$ given by $\tau_{3}$ in $\mathcal{B}$ and $C$ is $U_{3}=(6 / 20)=0,3$. Thus, the admission control executed during the negotiation phase at $t=2$ (in $\mathcal{B}$ ) and $t=3$ (in $C$ ) gives a positive response to its activation, being $U^{\mathcal{B}, C}=0.5 \leq 1$. Therefore, $\tau_{4}$ is activated for the first time at $t=8$ (see Fig. 4(b)).

The exact logs of the events happening inside the three agents during the system execution follow are detailed accordingly: $\mathcal{A}$ (Table 4), $\mathcal{B}$ (Table 5), and $C$ (Table 6):

\section{Conclusions}

This paper presented a comprehensive review and analysis of solutions empowering telerehabilitation. Particular emphasis has been given to agent-based systems, presenting their features, limitations, and formalizing the open challenges.

Physiotherapist needs and requirements for telerehabilitation have been presented and formalized. Furthermore, the needs still left unsatisfied by inadequate systems on the market, with respect to conventional non-technological practices, have been highlighted.

Elaborating on existing rehabilitation MAS, the identified strengths are the possibility of (i) easy scenario contextualization, (ii) facing uncertainties related to planning and problem solving, (iii) coordinating distributed sources of information, and sophisticated distributed controls. Beside these comproved positive features, limitations such as (i) incompatibility with real-time operating systems, (ii) impossibility of running agents in embedded devices, and (iii) neglect of timing concepts within the agent ecosystem, claimed the need for new contributions.

Thus, the most relevant new challenges identified in the MAS's future steps are: (i) implementing time-aware mechanisms into the agent internal scheduler, communication and negotiation protocols, (ii) coping with scarcity of resources, and (iii) implementing standard protocols for new communication means.

Finally, a practical case-study employing the proposed solutions has been analyzed. Such a practical example: (i) showed the mechanisms of the CBS scheduling algorithm operating as local scheduler in MAS, (ii) confirmed the crucial support provided by the CBS as local scheduler for a reservation-based negotiation protocol [69], and (iii) showed the capability of guaranteeing the fully compliance with the MAS standards [84].

\subsection{Future work}

According to Amatya et al. [19], rigorous studies are still needed for future research in appropriate outcome measurements, optimal intensity, frequency, and cost effectiveness of telerehabilitation intervention over a longer period of time. Thus, by tightly collaborating with professional physiotherapists and researchers, we aim at facing the identified new challenges bringing the multi-agent features at the sensing level. The first expected outcomes will primarily be a fully distributed and real-time compliant MAS for knee rehabilitation, to later be employed in clinical trials and deliver appropriate studies over an extended period of time.

\section{Appendix A. Questionnaire}

(1) Which human joints and limbs are your (physiotherapist) primary interest?

(2) What are the most typical causes/conditions? (e.g., pre-post-surgical, post-stroke, or just aging-related)

Concerning the joint-limbs, you mentioned in the first question:

(3) How are they treated along the four phases (acute, subacute, chronic, and maintenance)?

(4) Which body parts are involved in the rehabilitation practices?

(5) Which body parts must be (or should be) monitored?

Concerning the previous answers:

(6) Generally, and in your department, which are the most performed/required rehabilitative practices? (e.g., per body part-type \& n. Of test)

(7) Are they more frequently performed in ambulatory/hospital or a home/home-like environment? 
(8) In such practices, what is possible to observe? (e.g., extension, flexion, $n$. of repetitions, punctual accuracy)

(9) In such practices, what is not possible to observe? (e.g., pain, fatigue, accurate evolution trend)

(10) In such practices, what should and what should not the patient do? (e.g., regarding position, execution-speed)

(11) What are the most common errors typically performed by the patients? (e.g., compensation)

(12) What are the most common errors typically performed by the physiotherapists? (e.g., misreadings)

(13) What are the (human) patient limits (what should they perceive or understand, but cannot)?

(14) What are the (human) physiotherapist limits (what would you like, but you cannot perceive or understand)?

(15) Concerning the technological research, what do you feel is missing and needs to be implemented?

\section{References}

[1] Calvaresi D, Cesarini D, Sernani P, Marinoni M, Dragoni AF, Sturm A. Exploring the ambient assisted living domain: a systematic review. J Ambient Intell Humaniz Comput 2016:1-19.

[2] Buonocunto P, Giantomassi A, Marinoni M, Calvaresi D, Buttazzo G. A limb tracking platform for tele-rehabilitation. ACM Trans Cyber-Phys Syst 2018;2(4):30. https:// doi.org/10.1145/3148225.

[3] Cesarini D, Calvaresi D, Marinoni M, Buonocunto P, Buttazzo G. Simplifying telerehabilitation devices for their practical use in non-clinical environments. International conference on bioinformatics and biomedical engineering. 2015. p. 479-90.

[4] Hailey D, Roine R, Ohinmaa A, Dennett L. Evidence of benefit from telerehabilitation in routine care: a systematic review. J Telemed Telecare 2011;17(6):281-7.

[5] Dubovitskaya A, Calvaresi D, Schumacher MI. Essais cliniques multicentriques: transparence et contrôle de la qualité grâce à la blockchain et aux systèmes multiagents. Swiss Med Inform 2018;34.

[6] Fasola J, Mataric M. A socially assistive robot exercise coach for the elderly. J Human-Robot Interact 2013;2(2):3-32.

[7] Obdržálek Š, Kurillo G, Ofli F, Bajcsy R, Seto E, Jimison H, et al. Accuracy and robustness of kinect pose estimation in the context of coaching of elderly population. 2012 annual international conference of the IEEE engineering in medicine and biology society. 2012. p. 1188-93.

[8] Rodriguez AC, Roda C, González P, Navarro E. Contextualizing tasks in tele-rehabilitation systems for older people. International workshop on ambient assisted living. 2015. p. 29-41.

[9] Calvaresi D, Cesarini D, Marinoni M, Buonocunto P, Bandinelli S, Buttazzo G. Nonintrusive patient monitoring for supporting general practitioners in following diseases evolution. International conference on bioinformatics and biomedical engineering. 2015. p. 491-501.

[10] Mozaffarian D, Benjamin EJ, Go AS, Arnett DK, Blaha MJ, Cushman M, et al. Executive summary: heart disease and stroke statistics-2016 update: a report from the American heart association. Circulation 2016;133(4):447.

[11] Kairy D, Lehoux P, Vincent C, Visintin M. A systematic review of clinical outcomes, clinical process, healthcare utilization and costs associated with telerehabilitation. Disabil Rehabil 2009;31(6):427-47.

[12] Mutingi M, Mbohwa C. Developing multi-agent systems for mhealth drug delivery. Mobile health. Springer; 2015. p. 671-83.

[13] Morreale PA. Wireless sensor network applications in urban telehealth. 21st international conference on advanced information networking and applications workshops, 2007, AINAW'07, vol. 2. 2007. p. 810-4

[14] McNaull J, Augusto JC, Mulvenna M, McCullagh P. Multi-agent interactions for ambient assisted living. 2011 7th international conference on intelligent environments (IE). 2011. p. 310-3.

[15] Ayala I, Amor M, Fuentes L. Self-configuring agents for ambient assisted living applications. Person Ubiquit Comput 2013;17(6):1159-69.

[16] Hurtado C, Ramirez MR, Alanis A, Vazquez SO, Ramirez B, Manrique E. Towards a multi-agent system for an informative healthcare mobile application. KES international symposium on agent and multi-agent systems: technologies and applications. 2018. p. 215-9.

[17] Calvaresi D, Schumacher M, Marinoni M, Hilfiker R, Dragoni AF, Buttazzo G. Agentbased systems for telerehabilitation: strengths, limitations and future challenges. Agents and multi-agent systems for health care. 2017. p. 3-24.

[18] Buttazzo G. Hard Real-Time Computing Systems: Predictable Scheduling Algorithms and Applications vol. 24. Springer Science \& Business Media; 2011.

[19] Amatya B, Galea M, Kesselring J, Khan F. Effectiveness of telerehabilitation interventions in persons with multiple sclerosis: a systematic review. Multiple Scler Relat Disord 2015;4(4):358-69.

[20] Adibi S. Mobile Health: A Technology Road Map vol. 5. Springer; 2015.

[21] Krueger RA, Casey MA. Focus groups: a practical guide for applied research. Sage Publications; 2014.

[22] L. Beny, R. Griesmar, Device for producing continuous passive motion, US Patent 6 ,
325,770 (December 4 2001)

[23] Roda C, Rodríguez AC, López-Jaquero V, Navarro E, González P. A multi-agent system for acquired brain injury rehabilitation in ambient intelligence environments. Neurocomputing 2017;231:11-8.

[24] Calvaresi D, Appoggetti K, Lustrissimini L, Marinoni M, Sernani P, Dragoni AF, et al. Multi-agent systems' negotiation protocols for cyber-physical systems: results from a systematic literature review. Proceedings of 10 th international conference on agents and artificial intelligence 2018.

[25] Kitchenham B, Brereton P, Turner M, Niazi M, Linkman S, Pretorius R, et al. Refining the systematic literature review process-two participant-observer case studies. Empir Softw Eng 2010;15(6):618-53. https://doi.org/10.1007/s10664010-9134-8.

[26] Galster M, Weyns D, Tofan D, Michalik B, Avgeriou P. Variability in software systems - a systematic literature review. IEEE Trans Softw Eng 2014;40(3):282-306. https://doi.org/10.1109/TSE.2013.56.

[27] Korzeniowska-Kubacka I, Dobraszkiewicz-Wasilewska B, Bilińska M, Rydzewska F, Piotrowicz R. Two models of early cardiac rehabilitation in male patients after myocardial infarction with preserved left ventricular function: comparison of standard out-patient versus hybrid training programmes. Kardiologia Polska (Pol Heart J) 2011;69(3):220-6.

[28] Li KF. Smart home technology for telemedicine and emergency management. J Ambient Intell Humaniz Comput 2013;4(5):535-46.

[29] Carignan CR, Krebs HI. Telerehabilitation robotics: bright lights, big future? J Rehabil Res Dev 2006;43(5):695.

[30] Mikołajewska E, Mikołajewski D. Neurological telerehabilitation-current and po tential future applications. J Health Sci 2011;1(4):7-14.

[31] Bergmann J, McGregor A. Body-worn sensor design: what do patients and clinicians want? Ann Biomed Eng 2011;39(9):2299-312.

[32] Akdoğan E, Taçgın E, Adli MA. Knee rehabilitation using an intelligent robotic system. J Intell Manuf 2009:20(2):195-202.

[33] Chen J, Zhang X, Li R. A novel design approach for lower limb rehabilitation training robot. 2013 IEEE international conference on automation science and engineering (CASE). 2013. p. 554-7.

[34] de Abreu PF, Werneck VMB, da Costa RMEM, de Carvalho LAV. Employing multiagents in 3-d game for cognitive stimulation. XIII symposium on virtual reality). 2011. p. 73-8.

[35] Johansson T, Wild C. Telerehabilitation in stroke care - a systematic review. J Telemed Telecare 2011;17(1):1-6.

[36] Iarlori S, Ferracuti F, Giantomassi A, Longhi S. Rgbd camera monitoring system for Alzheimer's disease assessment using recurrent neural networks with parametric bias action recognition. IFAC Proc Vol 2014;47(3):3863-8.

[37] Cameirão MS, Pereira F, i Badia SB. Virtual reality with customized positive stimuli in a cognitive-motor rehabilitation task. 2017 international conference on virtual rehabilitation (ICVR). 2017. p. 1-7.

[38] Oliver M, González P, Montero F, Molina JP, Fernández-Caballero A. Smart computer-assisted cognitive rehabilitation for the ageing population. Ambient in telligence-software and applications - 7th international symposium on ambient intelligence (ISAmI 2016). 2016. p. 197-205.

[39] Fraile JA, Bajo J, Corchado JM, Abraham A. Applying wearable solutions in dependent environments. IEEE Trans Inf Technol Biomed 2010;14(6):1459-67.

[40] Smith ST, Talaei-Khoei A, Ray M, Ray P. Agent-based monitoring of functional rehabilitation using video games. Advanced computational intelligence paradigms in healthcare 5. 2010. p. 113-41.

[41] Cesarini D, Buonocunto P, Marinoni M, Buttazzo G. A telerehabilitation framework for lower-limb functional recovery. International conference on body area networks. 2014

[42] Cesarini D, Calvaresi D, Farnesi C, Taddei D, Frediani S, Ungerechts BE, et al Mediation: an embedded system for auditory feedback of hand-water interaction while swimming. Proc Eng 2016;147:324-9.

[43] Ungerechts B, Cesarini D, Wiebel V, Hermann T. Ears drive hands: sonification of liquid effects induced by aquatic space activities contributes to cognitive representation. 2015

[44] Vourvopoulos A, Liarokapis F. Evaluation of commercial brain-computer interfaces in real and virtual world environment: a pilot study. Comput Electric Eng 2014;40(2):714-29.

[45] Eriksson J, Mataric MJ, Winstein C. Hands-off assistive robotics for post-stroke arm rehabilitation. Proc. IEEE international conference on rehabilitation robotics (ICORR'05) 2005:21-4.

[46] Jacobs A, Timmermans A, Michielsen M, Vander Plaetse M, Markopoulos P. Contrast: gamification of arm-hand training for stroke survivors. CHI'13 extended abstracts on human factors in computing systems. 2013. p. 415-20.

[47] BAMA VJM, et al. Pilot study: computer-based virtual anatomical interactivity for rehabilitation of individuals with chronic acquired brain injury. J Rehabil Res Dev 2014;51(3):377.

[48] Miranda P, Aguilar J. A prototype of a multiagents system for a telemedicine environment. Eng Intell Syst Electric Eng Commun 2003;11(1):3-8.

[49] F. Bergenti, A. Poggi, Multi-agent systems for the application and employing of ehealth services.

[50] Mekuria DN, Calvaresi D, Schumacher M, Dragoni AF, Bromuri S. Event calculus agent minds applied to diabetes monitoring. Agents and multi-agent systems for health care: 10th international workshop, A2HC 2017. 2017. p. 40

[51] Roda C, Rodríguez A, López-Jaquero V, González P, Navarro E. A multi-agent system in ambient intelligence for the physical rehabilitation of older people. Trends in practical applications of agents, multi-agent systems and sustainability. 2015. p. 113-23.

[52] Mesa I, Sanchez E, Diaz J, Toro C, Artetxe A. Gocardio: a novel approach for 
mobility in cardiac monitoring. InImpact: J Innov Impact 2016;6(1):110.

[53] Teruel MA, Navarro E, González P. Towards an awareness interpretation for physical and cognitive rehabilitation systems. Ubiquitous computing and ambient intelligence: 10th international conference, UCAmI 2016. 2016. p. 121-32.

[54] Felisberto F, Costa N, Fdez-Riverola F, Pereira A. Unobstructive body area networks (ban) for efficient movement monitoring. Sensors 2012;12(9):12473-88.

[55] Rohling ML, Faust ME, Beverly B, Demakis G. Effectiveness of cognitive rehabilitation following acquired brain injury: a meta-analytic re-examination of cicerone et al.'s $(2000,2005)$ systematic reviews. Neuropsychology 2009:23(1):20.

[56] Li C, Rusák Z, Horváth I, Ji L. Validation of the reasoning of an entry-level cyberphysical stroke rehabilitation system equipped with engagement enhancing capabilities. Eng Appl Artif Intell 2016;56:185-99.

[57] Su C-J, Peng CW. Multi-agent ontology-based web 2.0 platform for medical rehabilitation. Expert Syst Appl 2012;39(12):10311-23.

[58] Brugués A, Bromuri S, Barry MÓ, Del Toro J, Mazurkiewicz MR, Kardas P, et al. Processing diabetes mellitus composite events in magpie. J Med Syst 2016;40(2):1-15.

[59] Liao J, Hu C, Guan G, Meng MQ-H. An extensible telemonitoring architecture based on mobile agent method. 2009 IEEE international conference on robotics and biomimetics (ROBIO). 2009. p. 1537-42.

[60] Lai JC, Woo J, Hui E, Chan W. Telerehabilitation-a new model for communitybased stroke rehabilitation. J Telemed Telecare 2004;10(4):199-205.

[61] Schein RM, Schmeler MR, Saptono A, Brienza D. Patient satisfaction with telerehabilitation assessments for wheeled mobility and seating. Assist Technol ${ }^{\circledR}$ 2010;22(4):215-22.

[62] Calvaresi D, Sernani P, Marinoni M, Claudi A, Balsini A, Dragoni AF, et al. A framework based on real-time os and multi-agents for intelligent autonomous robot competitions. 2016 11th IEEE symposium on industrial embedded systems (SIES) 2016:1-10. https://doi.org/10.1109/SIES.2016.7509407.

[63] Bellifemine FL, Caire G, Greenwood D. Developing Multi-Agent Systems With JADE vol. 7. John Wiley \& Sons; 2007.

[64] Evidence, Erika enterprise rtos, http://www.evidence.eu.com.

[65] Fipa A. Fipa ACL message structure specification. Foundation for Intelligent Physical Agents; 2004. [30.06.04]. http://www.fipa.org/specs/fipa00061/ SC00061G.html.

[66] Gomez C, Oller J, Paradells J. Overview and evaluation of bluetooth low energy: an emerging low-power wireless technology. Sensors 2012;12(9):11734-53.

[67] Alliance Z, et al. Zigbee specification. 2006.

[68] Dragoni AF, Sernani P, Calvaresi D. When rationality entered time and became a real agent in a cyber-society. Proceedings of the 3rd International Conference on Recent Trends and Applications in Computer Science and Information Technology,
RTA-CSIT 2018. 2018. p. 167-71.

[69] D. Calvaresi, M. Marinoni, A. Sturm, M. Schumacher, G. Buttazzo, The challenge of real-time multi-agent systems for enabling iot and cps, in proceedings of IEEE/WIC/ ACM International Conference on Web Intelligence (WI'17). https://doi.org/10. $1145 / 3106426.3106518$.

[70] Tisue S, Wilensky U. Netlogo: design and implementation of a multi-agent modeling environment. Proceedings of agent, vol. 2004 2004:7-9.

[71] Bousquet F, Bakam I, Proton H, Le Page C. Cormas: common-pool resources and multi-agent systems. International conference on industrial, engineering and other applications of applied intelligent systems. 1998. p. 826-37.

[72] S. Chen, A. Tang, P. Stephens, H.-b. H. Chen, Simulation of multi-agent based scheduling algorithms for waiting-line queuing problems, Challenge, New Mexico Supercomputing.

[73] Gutknecht O, Ferber J. The madkit agent platform architecture. Workshop on infrastructure for scalable multi-agent systems at the international conference on autonomous agents. 2000. p. 48-55.

[74] Collier N. Repast: an extensible framework for agent simulation. Univ Chicago's Soc Sci Res 2003;36:2003

[75] N. Minar, R. Burkhart, C. Langton, M. Askenazi, et al. The swarm simulation system: A toolkit for building multi-agent simulations.

[76] Grignard A, Taillandier P, Gaudou B, Vo DA, Huynh NQ, Drogoul A. Gama 1. 6: advancing the art of complex agent-based modeling and simulation. International conference on principles and practice of multi-agent systems. 2013. p. 117-31.

[77] Luke S, Cioffi-Revilla C, Panait L, Sullivan K. Mason: a new multi-agent simulation toolkit. Proceedings of the 2004 swarmfest workshop. 2004. p. 316-27.

[78] JADE - Programmer Manual, http://jade.tilab.com/doc/programmersguide.pdf [Accessed 15.05.17].

[79] Bordini RH, Hübner JF. Bdi agent programming in agentspeak using jason. Proceedings of the 6th international conference on computational logic in multiagent systems. 2005. p. 143-64.

[80] Liu CL, Layland JW. Scheduling algorithms for multiprogramming in a hard-realtime environment. J ACM (JACM) 1973;20(1):46-61.

[81] Pardo-Castellote G. Omg data-distribution service: architectural overview. Distributed computing systems workshops. 2003. p. 200-6.

[82] Winters JM, Wang Y, Winters JM. Wearable sensors and telerehabilitation. IEEE Eng Med Biol Mag 2003;22(3):56-65.

[83] Bergenti F, Poggi A. Multi-agent systems for e-health: recent projects and initiatives. 10th int. workshop on objects and agents 2009.

[84] Foundation for Intelligent Physical Agents Standard, http://www.fipa.org/ [Accessed 24.09.17]. 